\title{
Simulation-based optimization approach for vehicle allocation in a private transport service: A case study
}

\author{
Andrés Muñoz-Villamizar ${ }^{a *}$, Jairo R. Montoya-Torres ${ }^{b}$ and Carlos A. Moreno-Camacho ${ }^{b}$
}

${ }^{a}$ Escuela Internacional de Ciencias Económicas y Administrativas, Universidad de La Sabana, km 7 autopista norte de Bogotá, D.C., Chí, Cundinamarca, Colombia

${ }^{b}$ Grupo de investigacón en Sistemas Logísticos, Facultad de Ingeniería, km 7 autopista norte de Bogotá, D.C., Universidad de La Sabana, Chí, Cundinamarca, Colombia

\section{CHR O N I C L E A B T T A C T}

Article history:

Received: October 18, 2018

Received in revised format: No-

vember 28, 2018

Accepted: December 7, 2018

Available online:

December 7, 2018

Keywords:

Optimization

Simulation

City logistics

Urban transport systems

Service level

Waiting time

\begin{abstract}
Poor urban planning and traffic congestion lead to excessive delays in workers' transit times and decrease their quality of life, especially in emerging countries. Several medium and large companies have a need to hire a transport service for their staff. In this type of transportation system, there is a heterogeneous fleet of vehicles, which are assigned to a set of pre-defined routes. However, the total transport delay can be even greater if the private transportation system is inefficient or not controlled. The approach proposed in this study seeks to optimize the private transport service by defining the best allocation of its fleet to its routes. A mathematical model is proposed to minimize user wait times. This approach is validated using real data obtained from a transport company in Colombia. The results demonstrate the quantitative benefits that can be achieved when the proposed approach is implemented, represented by a considerable reduction in user wait times.
\end{abstract}

(C) 2019 by the authors; licensee Growing Science, Canada

\section{Introduction}

Urban transportation systems significantly influence the urban structure and lives of residents in many cities, while also directly stimulating the economy (Tamaki et al., 2016). With increasing population, the demand for transportation in many cities is also growing. An increasing amount of traffic is on roads, which in turn creates greater mobility-related problems such as congestion, air pollution, noise pollution, and accidents, particularly in city centers, where the level of human activity is high (Farahani et al., 2013). Congestion was estimated to cost on the order of $\$ 124$ billion in 2013 , and is projected to reach approximately $\$ 186$ billion by 2030 (Guerrini, 2014). Chen and Nozick (2016) noted that travelers in congested areas needed to allow an hour of travel time for a trip that should take only 20 min in free-flowing traffic conditions. Public transport and pedestrian and bicycle traffic can help reduce this decline in quality of life. In general, public transport facilities reduce the environmental load resulting from traffic congestion and exhaust gas emissions. However, ineffectively operated facilities do not

* Corresponding author. Tel.: +57 18615555 Ext. 21106

E-mail address: andres.munoz1@unisabana.edu.co (A. Muñoz-Villamizar) 
necessarily reduce the environmental load, and operators may not be able to run these facilities sustainably (Tamaki et al., 2016).

An urban passenger transport system is typically a large, open, complex system containing several subsystems. In such a complex system, small changes in societal, economic, or environmental factors can lead to enormous changes in urban passenger transport development and energy consumption and emissions (Liu et al., 2015). In recent years, modeling strategies for transport systems have been particularly focused on understanding transport decisions. These models have incorporated a new set of variables in addition to variables describing the journey cost and user incomes, ages, gender, and social class (Tironi, 2016). In most freight transportation systems, the system retains control over the route choice node operating protocol and other operating decisions. However, in most passenger service systems, the users exercise considerable control over their own routing. In these instances, design models must include user behavior models (Magnanti \& Wong, 1984). Consequently, quantitative analysis of behavioral characteristics in urban passenger transport has become an important area of investigation.

The scientific literature includes extensive reports of academic studies and real-life applications of transport problems using well-known approaches from Operations Research (OR). Research in transportation and logistics has not only produced increased knowledge measurable through academic results in papers published and conferences organized. In addition, having always been driven by real problems, this research has also produced models and algorithms that have been embedded in software packages used by companies in the private and public sectors (Speranza, 2016). These studies seek to guide governments and decision makers for the appropriate planning of public transport networks to control urban traffic movements to ensure mobility and mitigate related problems.

Therefore, this study focuses on the concept and development of a new methodology to facilitate private transportation decision-making. The present study includes an analysis of private transportation systems, considering user wait times. To this end, a statistical analysis is performed to characterize the studied system. Then, an actual situation is simulated with the parameters of the case. Finally, a simulation-based optimization is performed to allocate vehicles to minimize user wait times. The results of both scenarios are then compared.

The rest of this paper is organized as follows: an overview of related literature is presented in Section 2; Section 3 describes the problem under study in detail, and the proposed solution approach is presented in Section 4; an example application to a real case is also presented in Section 4; and finally, Section 5 presents some concluding remarks and suggests topics for future research.

\section{Literature Review}

There is a large volume of theoretical and empirical literature focused on the design of urban transportation networks. Comprehensive literature reviews are provided in Farahani et al. (2013) and DiazParra et al. (2016). Transportation and logistics problems have been studied extensively by researchers and practitioners in OR. OR has provided fundamental contributions to supply chain management and transportation problems, and further important contributions are expected in response to new research challenges (Speranza, 2016). Designing an optimal public transport system in an urban context is an evolving and highly complex problem. In particular, finding the best line structure for real cities has proved to be an NP-hard problem, and thus has been approached using heuristics and metaheuristics (Fielbaum et al., 2016).

In general terms, a transport problem determines how to move a resource from an initial to a desired location with the lowest possible cost. Different cost functions lead to different optimization problems (e.g., minimization of the total distance or total emissions). According to Diaz-Parra et al. (2016), the general parameters of transportation problems are the following: 
- Resources: elements that can be transported from sources to destinations (e.g., goods, machines, tools, people, cargo, money, etc.)

- Locations: points of supply or collection (e.g., depots, nodes, railway stations, bus stations, loading ports, seaports, airports, refueling depots, etc.).

- Transportation modes: methods for transporting resources to the desired locations (e.g., ships, trucks, trains, etc.); different transportation modes require different infrastructure, capacity, times, activities, and regulations.

The planning, design, and management of new transportation infrastructure to serve new towns or improve existing transportation structures are traditionally addressed in an Urban Transportation Network Design Problem (UTNDP). The UTNDP deals with a complete hierarchy of decision-making processes for transportation planning, and includes strategic, tactical, and operational decisions (Magnanti \& Wong, 1984). In this context, strategic decisions are long-term decisions related to the infrastructure of transportation networks, including both transit and road networks; tactical decisions are concerned with the effective utilization of infrastructure and resources in existing urban transportation networks; and operational decisions are short-term decisions, which are mostly related to traffic flow control, demand management, or scheduling problems (Farahani et al., 2013).

On the other hand, there are a vast number of human-queueing phenomena associated with public transport services, which are commonly observed on a daily basis. Queueing theory was originally developed to describe problems in telecommunication, but has since been applied to many other fields, such as traffic flow, biology, and supply chains (Arita \& Schadschneider, 2014). This theory characterizes queueing systems according to customer arrival patterns (e.g., Poisson), service patterns, queue discipline (e.g., first-come-first-served), the number of servers provided, the maximum allowable queue length, and the server configuration (e.g., in series). Limited attention has been paid specifically to queueing in transportation research and traffic engineering (Wang et al., 2014). Queueing models in transport systems thus represent a critical gap in knowledge.

In particular, to the best of the authors' knowledge, no previous study has discussed the allocation of vehicles in the transportation services of private companies. The problem investigated in this study seeks to address the optimization of user waiting times in private transport services. The proposed model uses a design of experiments approach as well as approaches from the OR literature.

\section{Problem description}

Buffers, queues, and queuing delays are prevalent at the nodes of a communication system. The users of transportation networks, however, spend relatively more time on the links of the system (Magnanti \& Wong, 1984). Such queues in public transport services are observed at airports, urban bus/train/tram/metro stations, entries to people movers (e.g. cable cars), etc.

Customers are often discouraged by long queues. They typically tend to join a queue only when a short wait is expected, or will join a queue but then depart if a further wait would be intolerable. Long wait times in queues lead to two problems: balking and reneging. With balking, some customers will choose not to join a queue upon their arrival, normally because the queue ahead is too long. On the other hand, with reneging, some customers will first join a queue but gradually lose patience, and will eventually leave the queue before receiving service if the wait is intolerable (Wang et al., 2014). As queueing or waiting for transport services is an unpleasant experience, there is significant interest in better understanding and modeling this problem for a variety of economic, productivity, and efficiency calculations. Therefore, a significant volume of literature has been published in recent years on queuing for public transport (Arita \& Schadschneider, 2014). However, this problem also occurs in several unstudied situations, such as the wait time for a transportation vehicle that must be fully filled to be routed. 
A particular situation occurs in the transport services of private corporations. For example, in the case in this study, there are several different routes without specific departure times. In each of these routes, the assigned vehicle will leave the initial location only once it is completely filled, i.e., there are no empty places in the vehicle. Once the vehicle is filled, another vehicle arrives to repeat the process (See Fig. 1). This situation leads to even longer waiting times, which are directly related to the arrival times of users and the size of the vehicles that must be filled prior to departure. To handle this problem, an initial solution is to allocate vehicles with smaller capacities to routes with lower frequencies of arrival times. However, as the complexity of the problem increases (i.e., more routes and more available vehicles), the possible vehicle-route combinations also increase exponentially, leading to a combinatorial optimization problem. As a consequence, it is important to investigate the real impact of optimization models for various real-industry fields like that described above.

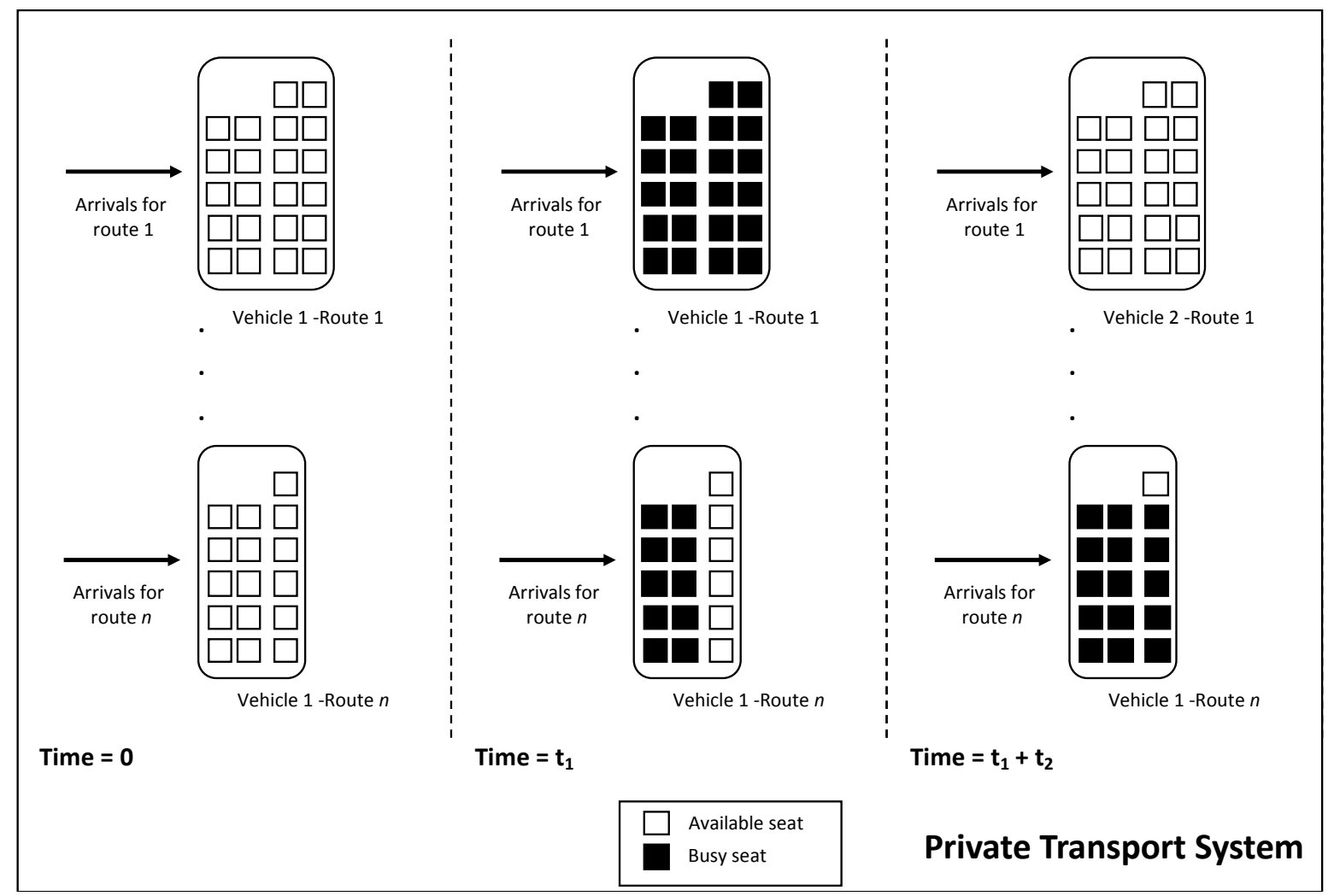

Fig. 1. Description of the private transport system in this study

\section{Solution approach}

As stated previously, this study seeks to optimize a private transport service by allocating vehicles using a simulation-based optimization approach. Simulation-based optimization methodologies have traditionally been employed for cost optimization in complex networks (Keramydas et al., 2017). However, the idea is to reduce user wait times as well as indirectly quantify the resulting reduction in operational costs and increase in efficiency of the transport system. According to Montoya et al. (2016), most of the academic literature in logistics research suggests that decision-makers in some industries largely prefer the use of simple and empirical solutions to deal with tactical and operational planning (i.e., medium- and short-term decisions). To achieve this, a four-step methodology is proposed (see Fig. 2). 


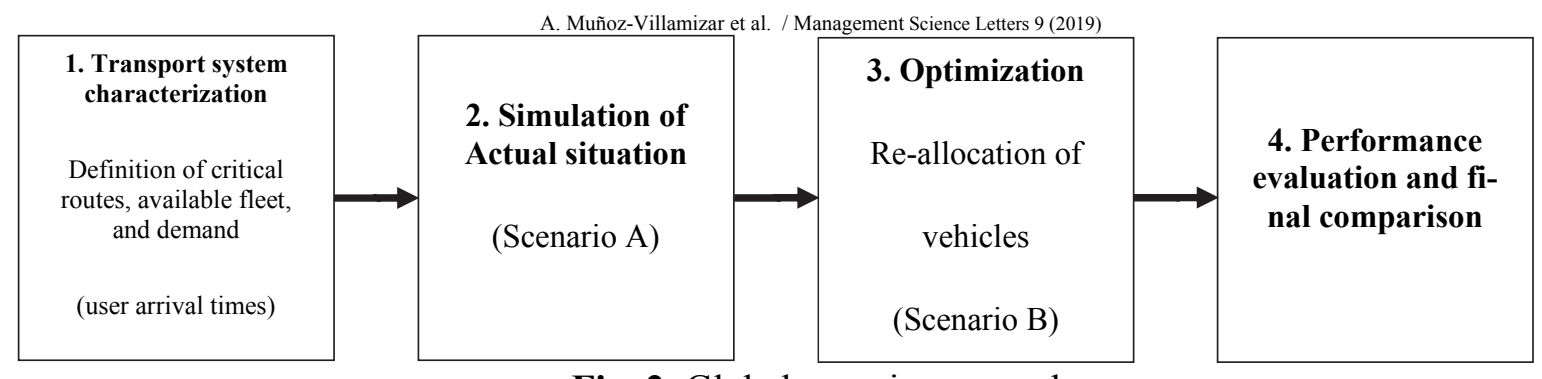

Fig. 2. Global generic approach

\subsection{Transport system characterization}

The objective of this first step is the identification of all elements in the case to be analyzed. The key elements are the number and schedule of routes, capacity of the available fleet, and demand (i.e., the arrival times of users to the system). However, other elements of interest may be included, such as the service level or atypical events (e.g., holidays).

Several statistical analyses are used in this characterization. First, to determine the most important routes in the system, a Pareto analysis is performed. This applies the $80 / 20$ rule, which makes the assumption that $20 \%$ of the routes serve $80 \%$ of all users of the system. The Pareto analysis reduces the system complexity, allowing for an understanding of the case under study without the need for huge efforts to obtain information.

For subsequent simulations, the user arrival times for each route must be defined. As many transportation systems are subject to substantial variability in demand, particularly due to morning and evening peak periods (Magnanti \& Wong, 1984), the main variables of the problem must be correctly evaluated. To do so, a multi-factor analysis of variance (ANOVA) is performed. Multi-factor ANOVA evaluates the relationship between variables to determine which of them influences the performance of the system. The user arrival time is selected as the main dependent variable. The independent variables, which represent the potential reasons for variation in the dependent variable are the type of route and time of day (i.e., the timeslot).

Finally, relevant statistical treatments, identified with the multi-factor ANOVA analysis, are fitted to a probability curve. In other words, a fit test is performed to calculate the probability distribution (e.g., Poisson, Erlang, lognormal, etc.) for the user arrival times with significant combinations between the type of route and time of day (e.g., Route 1-3:00 pm).

4.2 Simulation of an actual situation and simulation-optimization

In the second step of the proposed approach (simulation of the actual situation), a Monte Carlo simulation is carried out with the variables and components determined in the previous step. That is, the user arrival times are randomly generated according to the probability distribution previously identified for each relevant combination (e.g., Route 1-3:00 pm). Simulated data is then allocated to the vehicles according to the actual operation plan. This simulation model thus represents the actual performance of the system (Scenario A).

Then, for the third step (simulation-optimization), the user wait times in the system are optimized through the best allocation of vehicle types (i.e., vehicles with different capacities) to each route. For this, user arrival times are randomly generated according to the same probability distribution calculated in section 4.1. However, for this step, the allocation of vehicles to the defined routes is solved using the mixed-integer linear programming (MILP) model presented below. As different timeslots are evaluated (e.g., 3:00-4:00 pm, etc.), several instances of the same model are solved - one instance per timeslot. In other words, there is a different allocation of vehicles according to the available fleet for each timeslot. Note that this model assumes the user arrival times as an input parameter. Several binary 
variables are defined: $Y_{j k l}=1$ if vehicle $j$ completes trip $k$ with route $l$, and 0 otherwise; $W_{i l j k}=1$ if user $i$ of route $l$ is assigned to vehicle $j$ in trip $k$, and 0 otherwise. The positive variable $X_{j k}$ is defined as the departure time of vehicle $j$ for trip $k$. In addition, the following notation is defined:

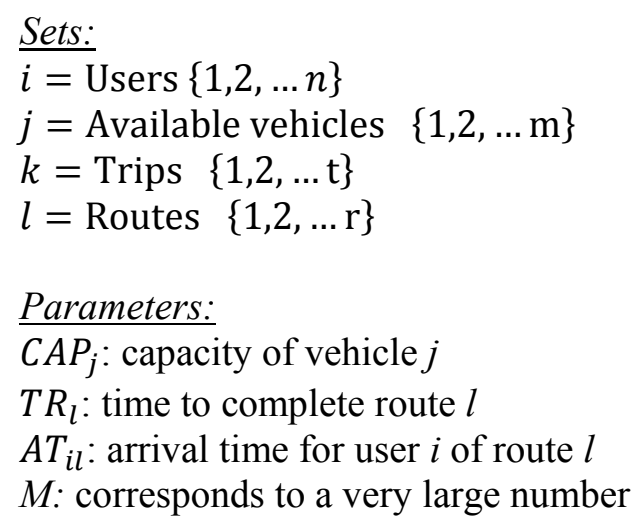

The mathematical model is as follows:

$$
\begin{aligned}
& \min \sum_{i} \sum_{l} \sum_{j} \sum_{k}\left(X_{j k}-W_{i l j k} * A T_{i l}\right) \\
& W_{i l j k} * A T_{i l} \leq X_{j k} \forall i, j, k, l \\
& X_{j k}+Y_{j k l} * T R_{l}-M\left(Y_{j k l+1}-1\right) \leq X_{j k+1} \forall j, k, l \\
& \sum_{l} Y_{j k l} \leq 1, \quad \forall j, k \\
& \sum_{j} \sum_{k} W_{i l j k}=1, \quad \forall i, l \\
& \sum_{i} W_{i l j k} \leq C A P_{j} * Y_{j k l}, \quad \forall j, k . l \\
& X_{j k} \geq 0, \quad \forall j, k \quad \forall i, j, k, l \\
& Y_{j k l}, W_{i l j k} \in\{0,1\}, \quad
\end{aligned}
$$

in which objective function (1) expresses the total wait time; constraint (2) ensures that each bus-route departure time is equal to the arrival time for the last assigned user; constraint (3) defines the trip continuity, i.e., a vehicle assigned to a route cannot be assigned to a different route until it returns to the departure station; constraint (4) guarantees that a bus can be assigned only one route per trip, i.e., the vehicle cannot go to two places at the same time; constraint (5) forces all clients to be assigned to a bus-route exactly once; constraint (6) establishes the capacity of each bus used on the routes; and finally, constraints (7) and (8) ensure variables only take positive and binary values, respectively. The results of this model for each instance lead to the optimized configuration for vehicle allocation, i.e., Scenario B.

\subsection{Performance evaluation and final comparison}

The final phase of the proposed approach consists of a comparison between the performance of the initial situation (Scenario A) and the situation after optimization (Scenario B). The user wait times are considered the objective function to be minimized and the main metric for comparing the scenarios. 
However, other metrics can also be evaluated, such as the service level, number of routes, vehicle utilization, and operational cost. It is important to note that the methodology proposed here is not a multi-objective approach. For the purposes of the current study, a single-objective optimization approach is carried out, with a posteriori evaluation of other metrics.

\section{Application and analysis of results}

To perform some numerical tests, the proposed framework was tested using real data from la Universidad de La Sabana, Colombia, as a quantitative sample. Note that this approach can be applied to other companies for a similar analysis.

La Universidad de La Sabana is a non-profit private institution of higher education, founded in Colombia in September 1979. It is an extensive campus ( 78 hectares) located in the town of Chía, which is 20 min from the country's capital (Bogotá). La Universidad de La Sabana is part of the group of institutions of higher education in Colombia with High Quality Institutional Accreditation. The University offers 75 academic programs: 22 undergraduate, 53 specializations, 26 Masters, and $3 \mathrm{PhDs}$, and has 362 full-time instructors (87\% with a PhD or Masters degree). La Universidad de La Sabana has 12,760 students: 8879 undergraduate, and 3881 graduate students (Universidad de La Sabana, 2017).

\subsection{Transport system characterization}

La Universidad de La Sabana outsources its transport system to a local company. On average, 2250 people use the university transport service daily (including students and university staff). The rest of the university population uses private cars, public transport, trains, or walking to reach the campus.

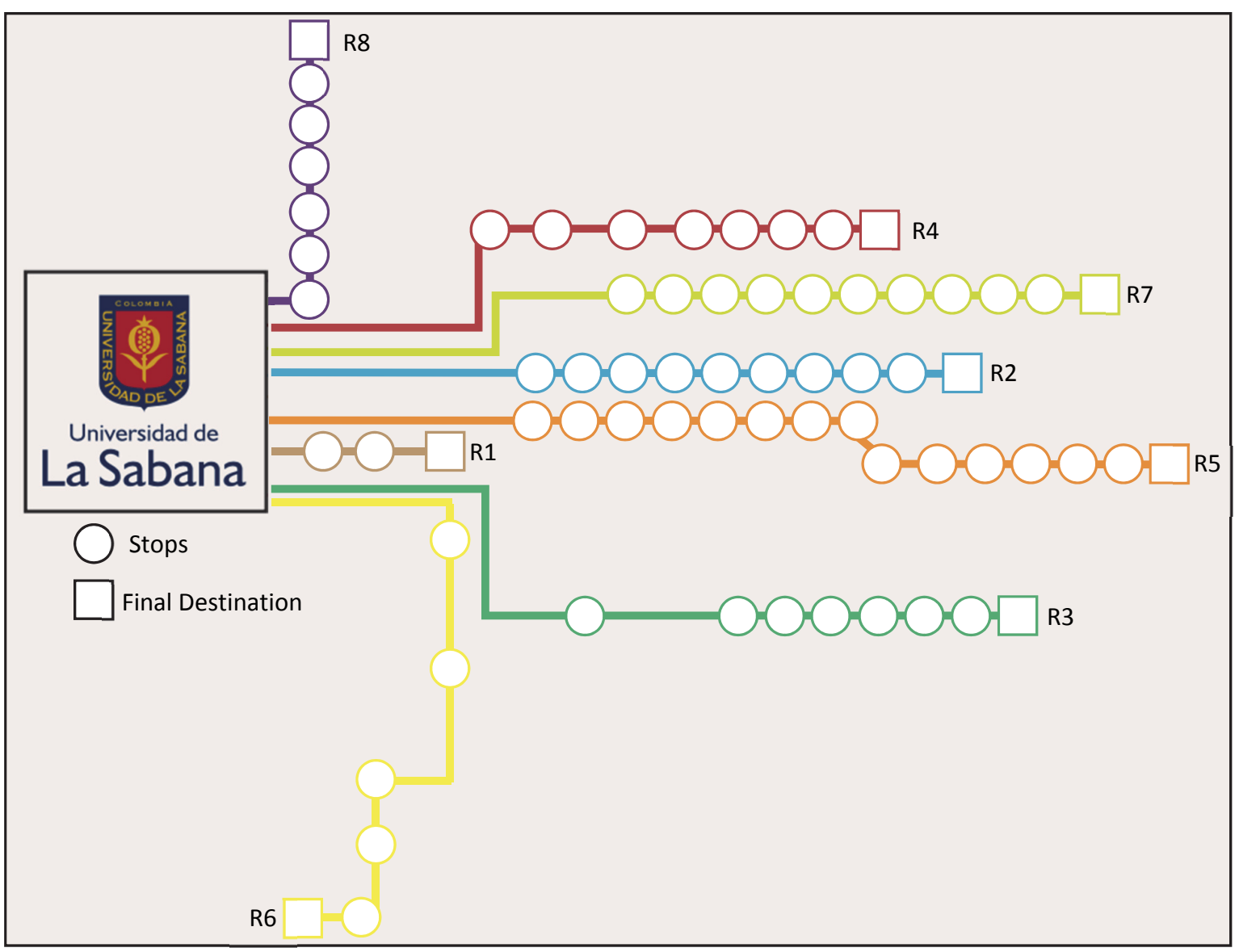

Fig. 3. Available transportation routes (Universidad de La Sabana, 2017) 
The outsourced transportation company has 35 permanent vehicles on campus, with capacities ranging from 14 to 44 people. In addition, 5 auxiliary vehicles are available for peak hours. There are 8 different routes that depart from the campus and travel through the main streets of Bogotá and to some nearby towns (see Fig. 3). These routes were defined based on market studies conducted by the company itself. Each route has a defined schedule and the company has a fixed allocation of vehicles based on the time of day. Applying an initial Pareto analysis to define the most important routes in the system reveals that $92 \%$ of users use 3 of the 8 available routes (see Fig. 4). These routes (Portal, Heroes, and Boyacá) are identified as R1, R2, and R3, respectively. Additionally, 6 timeslots were identified according to empirical company data, as summarized in Table 1 . These timeslots are identified herein as S1, S2, ... S6.

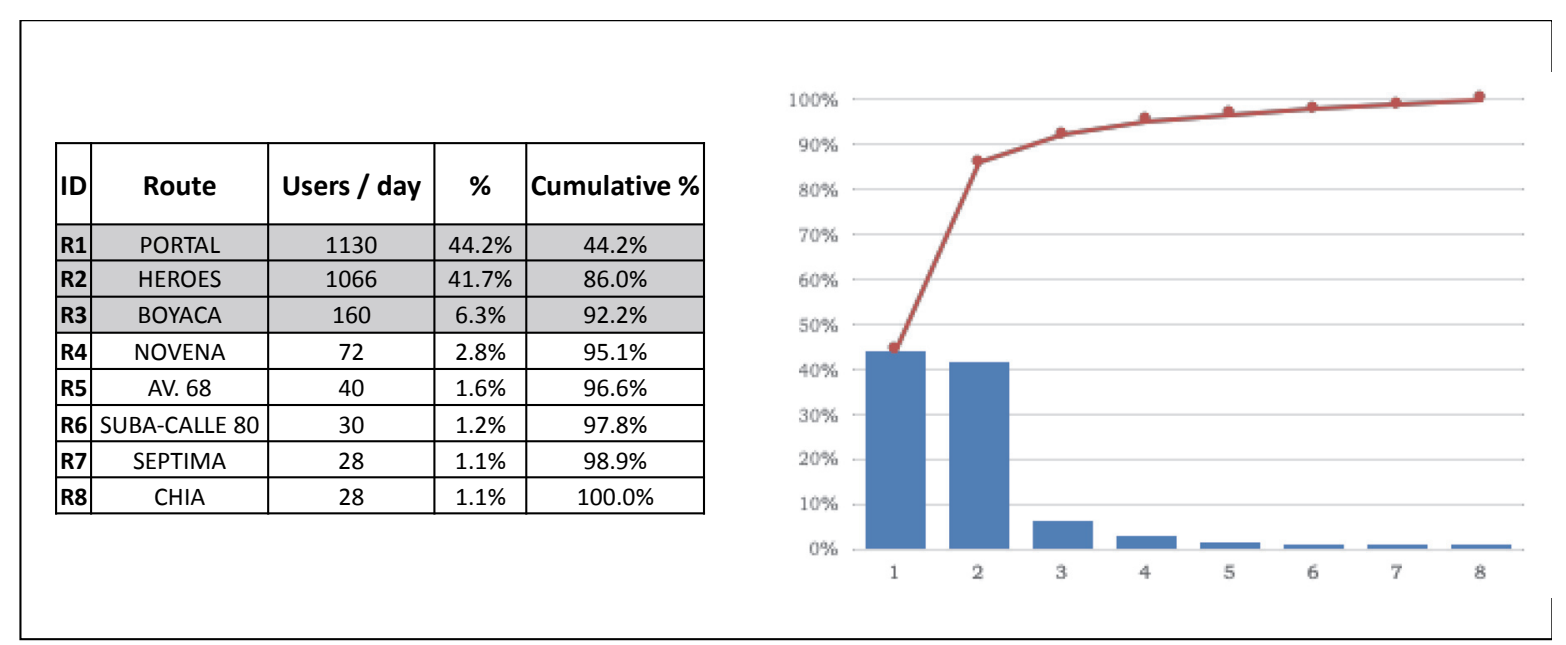

Fig. 4. Important routes identified using Pareto analysis (80/20 rule)

Table 1

Defined timeslots

\begin{tabular}{|c|c|c|}
\hline \multicolumn{3}{|c|}{ Timeslots } \\
\hline ID & From & To \\
\hline $\mathrm{S} 1$ & 9:00 a.m. & 11:00 a.m. \\
\hline $\mathrm{S} 2$ & 11:00 a.m. & 1:00 p.m. \\
\hline S3 & 1:00 p.m. & 3:00 p.m. \\
\hline S4 & 3:00 p.m. & 5:00 p.m. \\
\hline S5 & 5:00 p.m. & 7:00 p.m. \\
\hline S6 & 7:00 p.m. & 8:00 p.m. \\
\hline
\end{tabular}

Taking into account critical routes and timeslots, a two-factor ANOVA was carried out (see Fig. 5). Note that the combination of route R1 and time S6 has no data. As previously mentioned, each route has a defined schedule, and as a result some routes are not available at certain hours. On the other hand, as the time of day and route (i.e., the independent variables) were shown to significantly influence user arrival times (i.e., the dependent variable), the S5 timeslot (5:00-7:00 pm) was selected to reduce the complexity of the model.

Table 2

Daily users per timeslot

\begin{tabular}{ccc}
\hline Timeslot & Users / dav & $\%$ \\
\hline S5 & 474 & $27.3 \%$ \\
S4 & 471 & $27.1 \%$ \\
S3 & 329 & $19.0 \%$ \\
S2 & 217 & $12.5 \%$ \\
S6 & 133 & $7.7 \%$ \\
S1 & 112 & $6.4 \%$ \\
\hline Total & 1736 & $100.0 \%$ \\
\hline
\end{tabular}


Table 2 indicates that timeslot S5 contains $27 \%$ of the complete transport system. According to the initial Pareto analysis (i.e., $92.2 \%$ route coverage) and this new complexity reduction $(27.3 \%$ of daytime coverage), the model covers more than $25 \%$ of the whole system.

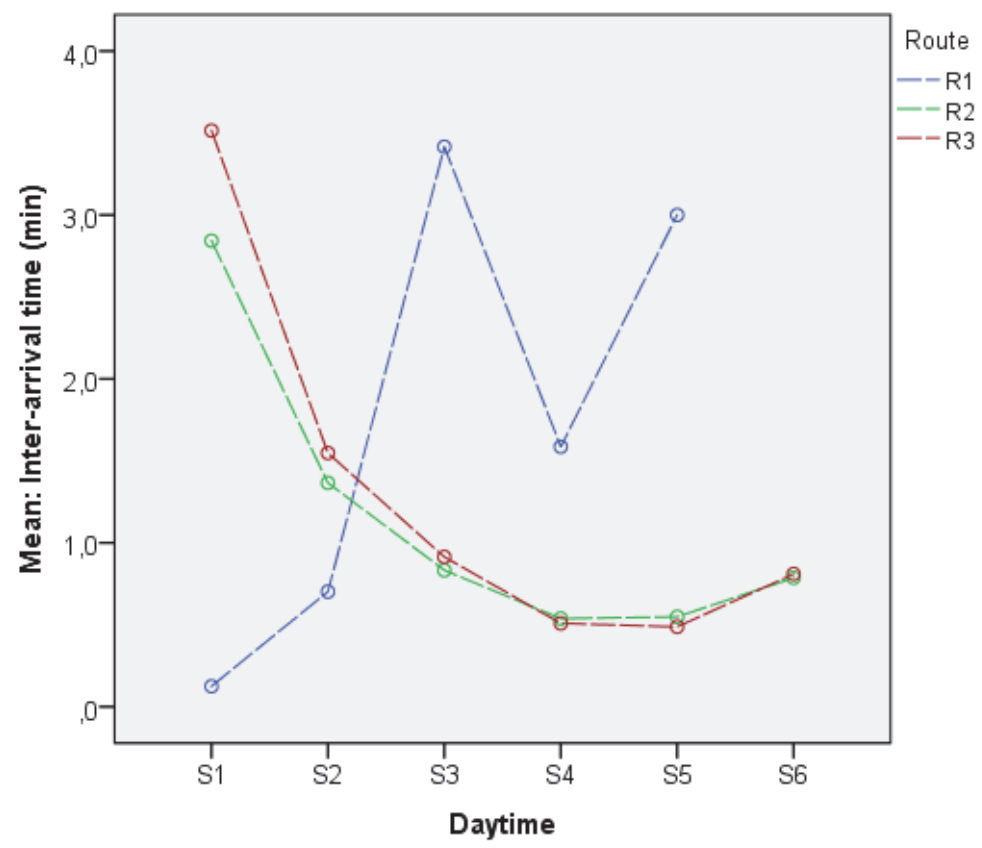

Fig. 5. Multi-factor ANOVA results

Finally, fit tests are performed to calculate the probability distribution of these relevant statistical treatments using commercially available software (i.e., Arena ${ }^{\circledR}$ ). Probability curves for the relevant treatments are shown in Fig. 6.

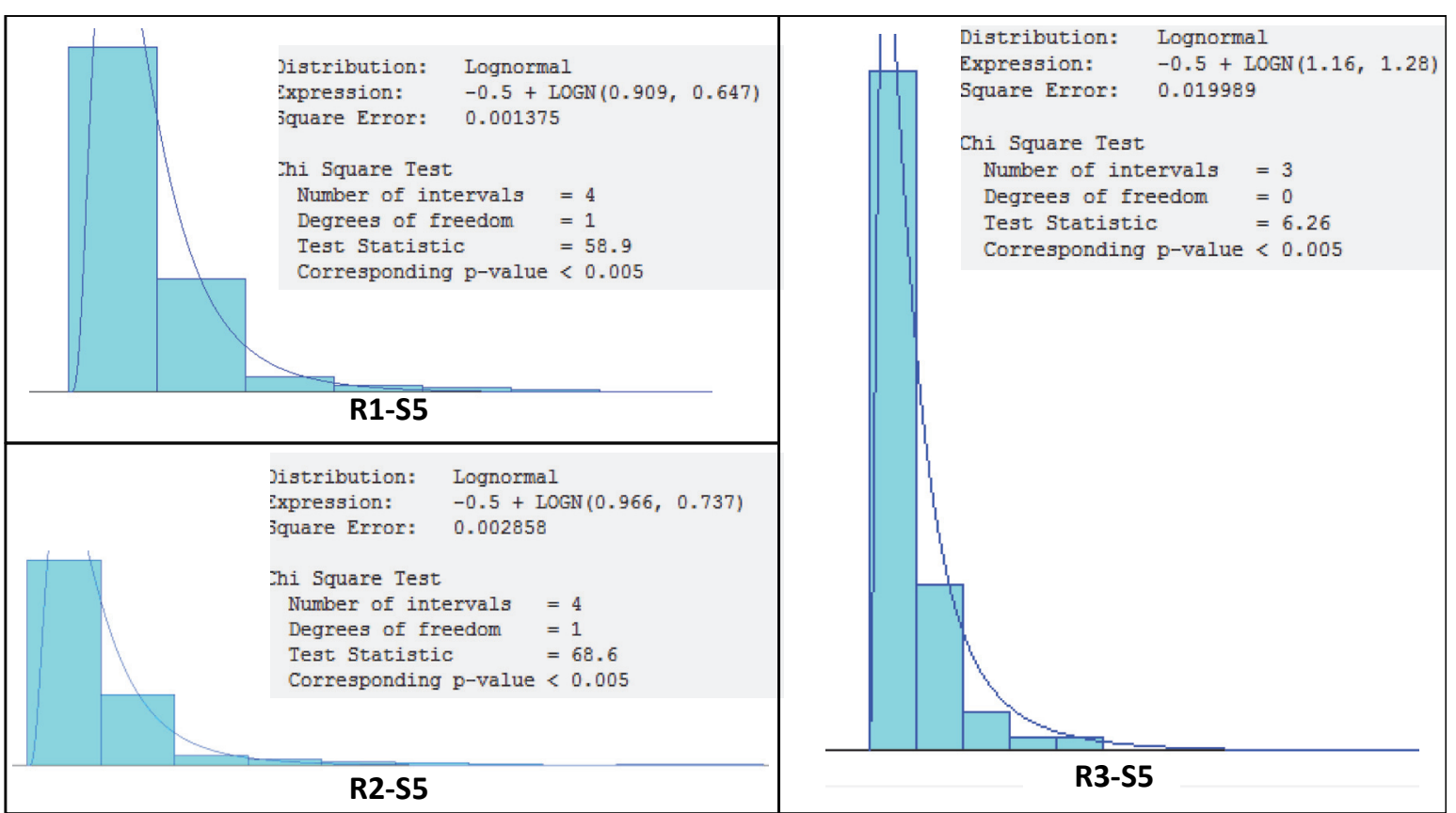

Fig. 6. Probability curves for treatments of routes R1, R2, and R3 with timeslot S5 
As shown in Fig. 6, the distribution of the inter-arrival times for every route fit most closely to a lognormal distribution. Given this result, a two-factor ANOVA was carried out using the natural logarithm of the inter-arrival time as the dependent variable, and the results are summarized in Table 3. The "Sig" column in Table 3 indicates the statistical significance level of the two-way interaction. The p-value of the routextimeslot row is less than 0.5 , which means that there is a statistically significant two-way interaction effect, as expected.

\section{Table 3}

ANOVA Results

Tests of Effects Between Subjects

Dependent Variable: Inter-Arrival Time

\begin{tabular}{lrrrrr}
\hline Source & $\begin{array}{c}\text { Type III Sum of } \\
\text { Squares }\end{array}$ & df & Mean Square & F & Sig \\
\hline Corrected model & $1025.395^{\text {a }}$ & 16 & 64.087 & 35.513 & 0.000 \\
Intercept & 1834.789 & 1 & 1834.789 & 1016.732 & 0.000 \\
Route & 15.948 & 2 & 7.974 & 4.419 & 0.012 \\
Timeslot & 154.929 & 5 & 30.986 & 17.17 & 0.000 \\
Route $\times$ Timeslot & 149.563 & 9 & 16.618 & 9.209 & 0.000 \\
Error & 15627.791 & 8660 & 1.805 & & \\
Total & 30234.114 & 8677 & & & \\
Corrected Total & 16653.186 & 8676 & & & \\
\hline
\end{tabular}

a. R Squared $=.062$ (Adjusted R Squared $=0.060$ )

5.2 Simulation of the actual situation and simulation-optimization

During actual operation, i.e., Scenario A, the transportation company has a fixed allocation of specific vehicles for each route. For routes R1, R2, and R3 during timeslot S5, there are 5 vehicles with different capacities available. As mentioned previously, the company has allocated these vehicles to each route according to its own empirical data. In contrast, the proposed model leads to an optimized allocation of the available fleet for each route, i.e., Scenario B. The simulation was executed with the selected treatments (i.e., routes R1, R2, and R3 with timeslot S5) for one month (25 working days). Results for the simulation of the actual situation (Scenario A) are presented in Table 4, while the results of the simulation-optimization model (Scenario B) are presented in Table 5.

Table 4

Results for the simulation of the actual situation

\begin{tabular}{|c|c|c|c|c|}
\hline \multicolumn{2}{|c|}{ Scenario A (Actual) } & \multicolumn{3}{|c|}{ Timeslot: 5:00 pm - 7:00 pm } \\
\hline Bus & Capacity & Pre-Assigned Route & Average & Average \\
\hline B1 & 21 & R1 & 42.16 & $5: 42 \mathrm{pm}$ \\
\hline B2 & 33 & $\mathrm{R} 2$ & 33.02 & $5: 33 \mathrm{pm}$ \\
\hline B3 & 33 & $\mathrm{R} 2$ & 61.50 & $6: 02 \mathrm{pm}$ \\
\hline B4 & 41 & $\mathrm{R} 3$ & 34.98 & $5: 35 \mathrm{pm}$ \\
\hline B5 & 41 & R3 & 62.64 & $6: 03 \mathrm{pm}$ \\
\hline
\end{tabular}

\section{Table 5}

Results with the optimization-simulation model

\begin{tabular}{ccccc}
\hline \multicolumn{2}{c}{ Scenario B } & \multicolumn{2}{c}{ Timeslot: 5:00 pm - 7:00 pm } \\
\hline Bus & Capacity & Pre-Assigned Route & $\begin{array}{c}\text { Average } \\
\text { "Filling” Time (min) }\end{array}$ & $\begin{array}{c}\text { Average } \\
\text { Departure Time }\end{array}$ \\
\hline B1 & 21 & R3 & 17.73 & $5: 18 \mathrm{pm}$ \\
B2 & 33 & R2 & 68.79 & $6: 09 \mathrm{pm}$ \\
B3 & 33 & R1 & 59.16 & $5: 59 \mathrm{pm}$ \\
B4 & 41 & R2 & 38.23 & $5: 38 \mathrm{pm}$ \\
B5 & 41 & R3 & 44.37 & $5: 44 \mathrm{pm}$ \\
\hline
\end{tabular}


Results for the routing with Scenario A and Scenario B (i.e., simulation of the actual situation and simulation-optimization of the problem, respectively) are presented in Fig. 7. The total time for Scenario A is $234.3 \mathrm{~min}$, while the total time for Scenario B is $228.27 \mathrm{~min}$. Although there is a reduction of only $6 \mathrm{~min}$, the simulation-optimization approach (Scenario B) was able to improve average user wait times by $13.96 \%$ compared to the simulation of the actual situation. In other words, the average wait time decreases from $17.04 \mathrm{~min} /$ user to $9.91 \mathrm{~min} /$ user, which is a reduction of $7.13 \mathrm{~min} /$ user. The total utilization of vehicles, total number of users transported, and thus the system income, are the same in both scenarios.

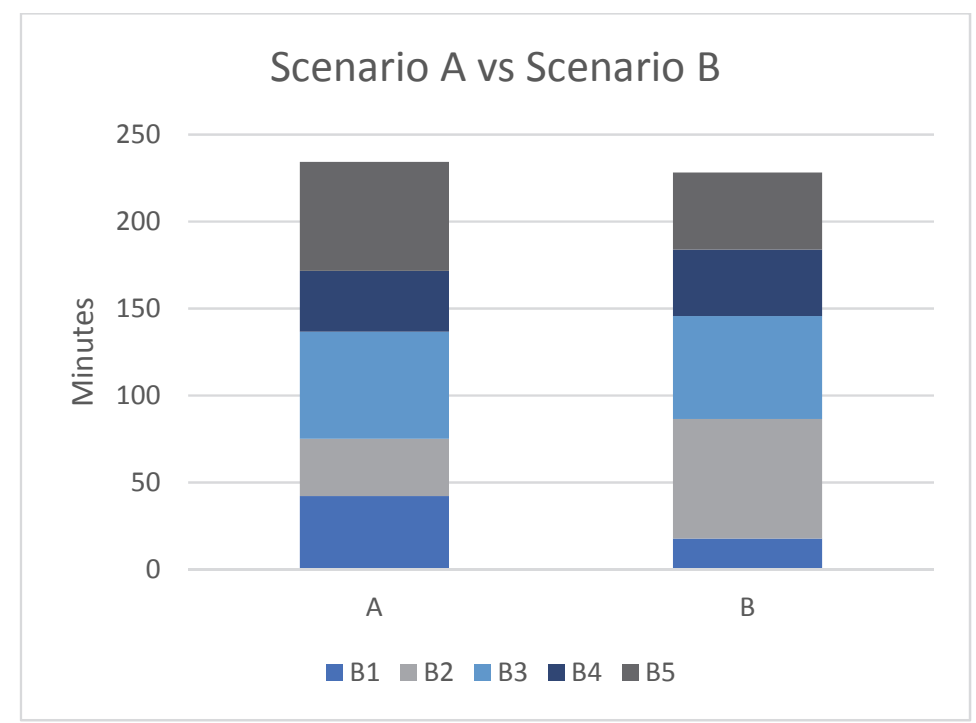

Fig. 7. Comparison of the simulated scenarios

\section{Conclusions and further research}

This paper proposed an approach for determining the allocation of vehicles in a private transport service based on a simulation-optimization model to minimize the user wait times. Computational tests were carried out using real data from the transport service at La Universidad de La Sabana, Colombia. Statistical tools such as Pareto analysis and multi-factor ANOVA were used to characterize the system. Additionally, the size of the studied system was reduced to decrease the model complexity, achieving a coverage of $25 \%$ of the transport system. The results demonstrate the quantitative benefits that can be achieved by implementing the proposed approach, represented by a reduction of almost $14 \%$ in user wait times in the optimized scenario.

The proposed approach can be used as a guideline for private decision-makers to improve the efficiency of their transport systems. The main contribution of this study is that user satisfaction could be improved without affecting the profitability of the system. To the best of the authors' knowledge, this is the first study that proposes an approach to improve the performance of a private transport system.

In real applications, problems could arise after the new policy is implemented, which presents an opportunity for further research. These problems may include a lack of information about routes, bus delays, service shortages in certain parts of the capital, increased journey times, and long queues for routes due to underestimation of the journey length. Other types of problems could arise when companies force employees to change payment methods. That is, using an electronic card instead of cash, which means remembering to charge the card with credit before getting on the bus. Finally, the complexity of the bus-route allocation problem leads to the design of approximate algorithms, primarily based on meta-heuristics. 


\section{Acknowledgments}

The authors would like to acknowledge the support received from AdP, the Special Patrimonial Fund at Universidad de La Sabana.

\section{References}

Arita, C., \& Schadschneider, A. (2014). The dynamics of waiting: The exclusive queueing process. Transportation Research Procedia, 2, 87-95.

Chen, R., \& Nozick, L. (2016). Integrating congestion pricing and transit investment planning. Transportation Research Part A: Policy and Practice, 89, 124-139.

Díaz-Parra, O., Ruiz-Vanoye, J.A., Bernábe Loranca, B., Fuentes-Penna, A., Barrera-Cámara, R.A. (2014). A survey of transportation problems. Journal of Applied Mathematics, Article ID 848129, 17 pages. doi: $10.1155 / 2014 / 848129$

Farahani, R.Z., Miandoabchi, E., Szeto, W.Y., Rashidi, H. (2013). A review of urban transportation network design problems. European Journal of Operational Research, 229(2), 281-302.

Fielbaum, A., Jara-Diaz, S., Gschwender, A. (2016) Optimal public transport networks for a general urban structure. Transportation Research Part B: Methodological, 94, 298-313.

Guerrini, F., 2015. Traffic Congestion Costs Americans \$124 Billion a Year, Report Says. Forbes. Available online at: http:/www.forbes.com/sites/federicoguerrini/2014/10/14/traffic-congestioncosts-americans-124-billion-a-year-report-says/. Accessed 14 December 2016.

Keramydas, C., Mallidis, I., Dekker, R., Vlachos, D., Iakovou, E. (2017). Cost and environmental tradeoffs in supply chain network design and planning: The merit of a simulation-based approach. Journal of Simulation, 11(1), 20-29.

Liu, X., Ma, S., Tian, J., Jia, N., Li, G. (2015). A system dynamics approach to scenario analysis for urban passenger transport energy consumption and $\mathrm{CO} 2$ emissions: A case study of Beijing. Energy Policy, 85, 253-270.

Magnanti, T.L., Wong, R.T. (1984), Network design and transportation planning: models and algorithms. Transportation Science, 18(1), 1-55.

Montoya-Torres, J. R., Solano-Charris, E. L., \& Muñoz-Villamizar, A. (2016). Assessing the Performance of Dispatching Policies for Hybrid Flowshop Manufacturing Systems. IFAC-PapersOnLine, 49(31), 109-113.

Speranza, M. G. (2018). Trends in transportation and logistics. European Journal of Operational Research, 264(3), 830-836.

Tironi, M., Palacios, R. (2016). Affects and urban infrastructures: Researching users' daily experiences of Santiago de Chile's transport system. Emotion, Space and Society, 21, 41-49.

Universidad de La Sabana. Población universitaria. Available at: http://www.unisabana.edu.co/unisabana/portafolio-institucional-2015-2016/secciones/poblacion-universitaria/ Last accessed: 18 January 2017. (In Spanish)

Wang, Y., Guo, J., Ceder, A.A., Currie, G., Dong, W., Yuan, H. (2014). Waiting for public transport services: Queueing analysis with balking and reneging behaviors of impatient passengers. Transportation Research Part B: Methodological, 63, 53-76

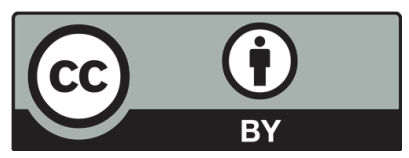

(C) 2019 by the authors; licensee Growing Science, Canada. This is an open access article distributed under the terms and conditions of the Creative Commons Attribution (CC-BY) license (http://creativecommons.org/licenses/by/4.0/). 